\title{
From laptops to supercomputers: a single highly scalable code base for spiking neuronal network simulations
}

\author{
Susanne Kunkel ${ }^{1,2,3^{*}}$, Maximilian Schmidt ${ }^{2}$, Jochen M Eppler ${ }^{2}$, Hans E Plesser $^{4}$, Jun Igarashi ${ }^{5}$, Gen Masumoto $^{6}$, \\ Tomoki Fukai ${ }^{5}$, Shin Ishii ${ }^{7}$, Abigail Morrison ${ }^{1,2,3,8}$, Markus Diesmann ${ }^{1,2,3,9}$, Moritz Helias ${ }^{2,5}$ \\ From Twenty Second Annual Computational Neuroscience Meeting: CNS*2013 \\ Paris, France. 13-18 July 2013
}

Over the last couple of years, supercomputers such as the Blue Gene/Q system JUQUEEN in Jülich and the K computer in Kobe have become available for neuroscience research. These massively parallel systems open the field for a new class of scientific questions as they provide the resources to represent and simulate brain-scale networks, but they also confront the developers of simulation software with a new class of problems. Initial tests with our neuronal network simulator NEST [1] on JUGENE (the predecessor of JUQUEEN) revealed that in order to exploit the memory capacities of such machines, we needed to improve the parallelization of the fundamental data structures. To address this, we developed an analytical framework [2], which serves as a guideline for a systematic and iterative restructuring of the simulation kernel. In December 2012, the $3^{\text {rd }}$ generation technology was released with NEST 2.2, which enables simulations of $10^{8}$ neurons and 10,000 synapses per neuron on the $\mathrm{K}$ computer [3].

Even though the redesign of the fundamental data structures of NEST is driven by the demand for simulations of interacting brain areas, we do not aim at solutions tailored to a specific brain-scale model or computing architecture. Our goal is to maintain a single highly scalable code base that meets the requirements of such simulations whilst still performing well on modestly dimensioned lab clusters and even laptops.

Here, we introduce the $4^{\text {th }}$ generation simulation kernel and describe the development workflow that yielded

\footnotetext{
* Correspondence: kunkel@fz-juelich.de

${ }^{1}$ Simulation Laboratory Neuroscience - Bernstein Facility Simulation and Database Technology, Institute for Advanced Simulation, Jülich Aachen Research Alliance, Jülich Research Centre, Germany

Full list of author information is available at the end of the article
}

the following three major improvements: the self-collapsing connection infrastructure, which takes up significantly less memory in the case of few local targets, the compacted node infrastructure, which causes only negligible constant serial memory overhead, and the reduced memory usage of synapse objects, which does not affect the precision of synaptic state variables. The improved code does not compromise on the general usability of NEST and will be merged into the common code base to be released with NEST 2.4. We show that with the $4 \mathrm{~g}$ technology it will be possible to simulate networks of $10^{9}$ neurons and 10,000 synapses per neuron on the $\mathrm{K}$ computer.

\section{Acknowledgements}

Partly supported by the early access to the K computer at the RIKEN Advanced Institute for Computational Science, by the VSR computation time grant JINB33 on the JUGENE and JUQUEEN supercomputers in Jülich, the Alliance on Systems Biology, Initiative and Networking Fund and Portfolio theme SMHB of the Helmholtz Association, the Jülich-Aachen Research Alliance (JARA), the Next-Generation Supercomputer Project of MEXT, EU Grant 269921 (BrainScaleS), Research Council of Norway Grant 178892N30 (eNeuro) and access to NOTUR supercomputing facilities. All network simulations carried out with NEST (http://www.nest-initiative.org).

\section{Author details}

Simulation Laboratory Neuroscience - Bernstein Facility Simulation and Database Technology, Institute for Advanced Simulation, Jülich Aachen Research Alliance, Jülich Research Centre, Germany. ${ }^{2}$ Institute of Neuroscience and Medicine (INM-6) and Institute for Advanced Simulation (IAS-6), Jülich Research Centre and JARA, Germany. ${ }^{3}$ Bernstein Center Freiburg, Albert-Ludwig University of Freiburg, Germany. ${ }^{4}$ Department of Mathematical Sciences and Technology, Norwegian University of Life Sciences, Aas, Norway. ${ }^{5}$ Laboratory for Neural Circuit Theory, RIKEN Brain Science Institute, Wako, Japan. ${ }^{6}$ High-Performance Computing Team, RIKEN Computational Science Research Program, Kobe, Japan. Integrated Systems Biology Laboratory, Department of Systems Science, Graduate School of Informatics, Kyoto University, Japan. ${ }^{8}$ Institute of Cognitive Neuroscience, Faculty of Psychology, Ruhr-University Bochum, Germany. ${ }^{9}$ Medical Faculty, RWTH University, Aachen, Germany. 


\section{References}

1. Gewaltig MO, Diesmann M: NEST (NEural Simulation Tool). Scholarpedia 2007, 2(4):1430.

2. Kunkel S, Potjans TC, Eppler JE, Plesser HE, Morrison A, Diesmann M: Meeting the memory challenges of brain-scale simulation. Front Neuroinform 2012, 5:35.

3. Helias M, Kunkel S, Masumoto G, Igarashi J, Eppler JE, Ishii S, Fukai T, Morrison A, Diesmann M: Supercomputers ready for use as discovery machines for neuroscience. Front Neuroinform 2012, 6:26.

doi:10.1186/1471-2202-14-S1-P163

Cite this article as: Kunkel et al:: From laptops to supercomputers: a single highly scalable code base for spiking neuronal network simulations. BMC Neuroscience 2013 14(Suppl 1):P163.

Submit your next manuscript to BioMed Central and take full advantage of:

- Convenient online submission

- Thorough peer review

- No space constraints or color figure charges

- Immediate publication on acceptance

- Inclusion in PubMed, CAS, Scopus and Google Scholar

- Research which is freely available for redistribution

Submit your manuscript at www.biomedcentral.com/submit
Ciomed Central 